\title{
Implementing person-centered communication in diabetes care: a new tool for diabetes care professionals
}

This article was published in the following Dove Press journal: Patient Preference and Adherence

\section{Ulla Connor' \\ Lucina Kessler ${ }^{2}$ \\ Mary de Groot ${ }^{3}$ \\ Robert Mac Neill ${ }^{4}$ \\ Robert Sandy ${ }^{5}$ \\ 'Department of English, Indiana University-Purdue University Indianapolis, Indianapolis, IN, USA; \\ ${ }^{2}$ Columbus Regional Health Hospital, Columbus, IN, USA; ${ }^{3}$ Diabetes \\ Translational Research Center, Indiana University School of Medicine, Indianapolis, IN, USA; ${ }^{4}$ CoMac Analytics, Inc., Indianapolis, IN, USA; ${ }^{5}$ Department of Economics, Indiana University-Purdue University Indianapolis, Indianapolis, IN, USA}

\section{Video abstract}

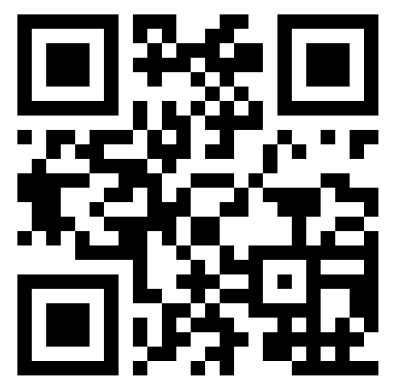

Point your SmartPhone at the code above. If you have a $Q R$ code reader the video abstract will appear. Or use: https://youtu.be/Q]nOXmBzfll

Correspondence: Ulla Connor Department of English, Indiana University-Purdue University Indianapolis, 425 University Blvd., Room I33, Indianapolis, IN 46202, USA

Tel + I $317278244 \mid$

Fax + I 3172742525

Email uconnor@iupui.edu
Purpose: This study tested the clinical implementation of the CoMac Communication System, an empirically validated tool for individualized Diabetes Self-Management Education and Support (DSMES). This system provides immediate feedback and guidance to health care providers (HCPs) to facilitate speaking with persons with type 2 diabetes mellitus in language reflecting patients' own worldviews and health beliefs.

Patients and methods: This 6-month implementation science study at an accredited diabetes care clinic in a Midwestern US hospital was conducted in two phases. Phase I consisted of CoMac implementation, qualitative interviews with HCPs, and evaluation of clinic flow among the diabetes education team. Seventy-two participants received CoMac's linguistically tailored patient-centric communication; a control group of 48 did not receive this intervention. In Phase II, glycosylated hemoglobin A1c (HbA1c) levels from the first visit to the follow-up visit for each group were compared.

Results: Interviews conducted during Phase I suggested that the system can be successfully implemented into DSMES practice. Knowing individual psychosocial profiles and participants' language use allowed for more effective patient counseling. In Phase II, multiple regression analysis with $\mathrm{HbAlc}$ change as the dependent variable showed that the key variable of interest, treated with the CoMac intervention, had a one-tailed $t$-value of -1.81 , with a statistically significant probability value of 0.037 .

Conclusion: Findings suggest that use of the CoMac System by diabetes care professionals has the potential for improved patient health outcomes. Patients receiving the CoMac intervention showed significantly improved HbA1c levels, suggesting that this approach has great promise for effective DSMES management.

Keywords: patient language, linguistics, diabetes self-management, clinical implementation

\section{Plain language summary}

The CoMac Communication System is an online tool that provides immediate feedback and guidance to health care providers (HCPs) to help them communicate more effectively with patients who have chronic illnesses. In short, the CoMac system provides HCPs with language that reflects patients' own worldviews and health beliefs. In the current study, we tested the adaptability of the CoMac system to an existing diabetes clinical practice, and we analyzed the A1c levels (a standard measurement for assessing the severity of the condition) over time of patients with type 2 diabetes whose providers used the CoMac tools versus those who did not use the tools. Interviews with the diabetes clinicians revealed the efficacy of the CoMac tools in providing more effective patient counseling. We found a statistically greater decline in Alc values over time in the 
patients with diabetes in our study who received the CoMac intervention when compared to those patients in our study who did not receive the CoMac treatment. Our findings suggest that the patient-centered CoMac approach has great promise for better adherence and health outcomes in patients with type 2 diabetes.

\section{Introduction}

Approximately 30.3 million people in the US already have diabetes, and 84.1 million are calculated to have increased risk for type 2 diabetes mellitus. ${ }^{1}$ Diabetes has become one of the costliest medical conditions in the US, with the total estimated cost of diagnosed diabetes in 2017 at US\$327 billion. $^{2}$ A significant contributor to the problem is poor adherence to healthy behaviors. At the heart of that issue is the patient's engagement in diabetes self-management. The American Diabetes Association (ADA) Standards of Medical Care in Diabetes 2019 recommended that treatment strategies should be "tailored to individual needs and preferences." 3 The 2017 National Standards for Diabetes Self-Management Education and Support (DSMES) also highlights the need to focus on patient motivation and barriers to self-management. ${ }^{1}$ The standards call for individualizing self-management education and support: "The DSMES service must be designed using person-centered care practices, in collaboration with the participant, focusing on the participant's priorities." 1

In 2001, the Institute of Medicine (IOM) identified a chasm in health care that led to six aims for improvement, namely that health care must be safe, effective, patientcentered, timely, efficient and equitable. ${ }^{4}$ Narrowing this gap considerably, significant improvements have been made in clinical information systems over the past two decades to support population management efforts. Language changes in policy and procedure have shifted to a patient-centric focus that includes individual culture, social context, respect, and active patient participation in care decisions. However, the dramatic increase in type 2 diabetes mellitus prevalence and subsequent burden magnifies the continued need for clinically applicable communication tools.

Certainly, the role of language in diabetes care and education is recognized as a key component of effective DSMES. The 2017 National Standards for DSMES acknowledge that language is the principle vehicle for sharing knowledge and creating understanding and that language is at the very core of attitude change and social perceptions. They emphasize that language has an impact on motivation, behaviors, and outcomes and recommend encouraging collaborative messages for enhancing patient engagement and empowerment. Specifically, the recommendation is for health care providers (HCPs) to use language that is nonjudgmental, free from stigma, respectful, and person-centered:

The time has come to reflect on the language of diabetes and share insights with others. Messages of strength and hope will signify progress toward the goals of eradicating stigma and considering people first. ${ }^{1}$

The Standards of Care 2019 go even further, urging providers to adopt a "patient-centered communication style" that "elicits patient preferences and beliefs," among other recommendations, toward achieving optimal health outcomes. $^{3}$ However, practical and feasible clinical patient-centered communication tools are limited in current health care settings. Indeed, there are few empirically validated communication tools that have been designed to assist diabetes educators and other health professionals to tailor communication with people with diabetes that meets patients at their current level of diabetes engagement and health beliefs about diabetes.

In the present study, we report a pilot implementation of an innovative language-centered communication tool, the CoMac Communication System. The CoMac System starts with the inherent assumption that language of the patient reveals his/her beliefs, worldviews, and orientation to self-management. ${ }^{5-9}$ Through understanding the person's orientation through language, this approach provides the clinician with tools for individualized advice and person-centered communication.

Prior linguistically based health communication research reveals that individuals' views of self and world are reflected in the language they use to talk about their health and self-management. These language patterns and styles of talk can be used to understand and explain individuals' attitudes, motivations, and barriers with regards to their health management. ${ }^{5-10}$

The CoMac Descriptor ${ }^{\mathrm{TM}}$, a 12-question survey questionnaire, categorizes patient responses to questions that evaluate health beliefs, locus of control, affect and personal agency into eight different clusters. These results are then summarized, and tools are provided to clinicians designed to individualize the communication with patients in a way that directly addresses the motivation 
and barriers revealed by the cluster. The patients complete the Descriptor survey questionnaire online, and the two subsequent communication tools, Points of Emphasis $^{\mathrm{TM}}$ and Linguistic Cues ${ }^{\mathrm{TM}}$, are immediately emailed to the HCP.

\section{Research questions}

The present study applied the CoMac System to the care of patients with type 2 diabetes mellitus, as part of a diabetes care clinic's ongoing patient education and management. The following questions were explored.

1. Can the CoMac System's tools be adapted for use by diabetes health care providers in an outpatient diabetes care setting?

2. What impact does the CoMac System have on the reduction of patient glycosylated hemoglobin Alc (HbA1c) levels compared to standard treatment?

\section{Materials and methods}

\section{Setting}

The study, which ran from January 2016 to December 2016, was conducted in a diabetes care clinic located in a Midwestern US health system. With a 100 -year history serving 10 counties in the state, the network consists of a 225-bed not-for-profit hospital with over 2100 employees, 225 providers on medical staff, and 250 volunteers. Its diabetes education services are accredited by AADE/DEAP, the American Association of Diabetes Educators Diabetes Education Accreditation Program.

The outpatient diabetes team in the study consisted of a nurse, dietitian, and a community health worker. All three were familiar with the CoMac approach, having participated in the CoMac research team's feasibility study by Bartlett Ellis et al. $^{8}$

\section{Population}

Of the total 386 patients seen by the diabetes team in the study period, 72 patients met all the inclusion criteria set for the intervention sample. Exclusion criteria included: gestational diabetes diagnosis, pregnancy diagnosis, any existing patient attending annual follow-up not new to service, and $\mathrm{HbAlc}$ measurements not performed by the participating care clinic's lab. The 72 patients meeting inclusion criteria had: 1) completed an initial assessment with the CoMac segmentation survey questionnaire, 2) completed goal settings, 3) had initial- and post-program $\mathrm{HbA1c}$ within at least 90 days between the measurements, and 4) attended one or more follow-up visits for DSMES.

The control group consisted of 48 patients who received the clinic's standard DSMES, without the use of the CoMac tools. This control group emerged due to the occasional unavailability of the iPads used to provide the survey and CoMac messaging. The unavailability generated a natural experiment because neither patient characteristics nor staff choice affected availability. Below, we examine statistically whether the control and intervention groups could have been equivalent to random draws from a common population.

Patient sample characteristics are shown in Table 1. There was no significant statistical difference between the CoMac intervention and control groups in three of the four available characteristics: age, gender, and starting weight. The average age for the intervention group was 61.5 years; the average for the control group was 62.4 $(t=0.60, P=0.720)$. A Chi-square test was used for the gender variable because that test was appropriate for a two-by-two contingency table of gender versus treatment status. The test of the null hypothesis that proportions of males and females across the intervention and control groups are equal yields a Chi-square test statistic of 0.022 , with a $P$-value of 0.881 . The starting weight for the intervention group was $224.7 \mathrm{lbs}$, and $221.7 \mathrm{lbs}$ for

Table I Baseline characteristics

\begin{tabular}{|l|l|l|l|l|}
\hline Characteristic & & CoMac intervention (n=72) & Control (n=48) & $P$-value \\
\hline Age, mean (SD), years & & $61.5(I 3.0)$ & $62.4(13.0)$ & 0.720 \\
Start weight, mean (SD), Ibs & & $224.7(48.6)$ & $221.7(74.7)$ & 0.792 \\
Start HbAlc, mean (SD) & $9.0(2.1)$ & $8.2(1.4)$ & 0.015 \\
Gender, n (\%) & & $34(47.2)$ & $22(45.8)$ & $0.88 \mid$ \\
& & $38(52.8)$ & $26(54.2)$ & \\
& Male & Female & & \\
\hline
\end{tabular}

Abbreviations: Ibs, pounds; HbAlc, glycosylated hemoglobin Alc. 
the control group $(t=-0.26, P=0.792)$. There was a statistically significant difference between the starting $\mathrm{HbAlc}$ between the two groups: $9.0 \%(75 \mathrm{mmol} / \mathrm{mol})$ for the intervention group and $8.2 \%(66 \mathrm{mmol} / \mathrm{mol})$ for the control group ( $t=-2.47, P=0.015)$.

The study did not require IRB review, as it was conducted in the interest of quality improvement to the clinic process. There was no stipend for participation in either the intervention or control group, and the clinic staff conducted the research without remuneration. The CoMac tools were provided by CoMac Analytics, Inc., to the clinic free of charge.

\section{CoMac system tools}

\section{CoMac descriptor survey}

In our previous research, ${ }^{5}$ a linguistic coding system was developed to analyze the actual words that people with type 2 diabetes mellitus use to describe their disease and its selfmanagement. The research identified linguistic indicators in the way patients talk that include agency (high/takes charge, low/does not take charge), affect (positive/upbeat, negative/ downbeat), and control orientation (internal/looks to self for directions, external/looks to others for directions). The linguistic features of these three well-studied psychosocial constructs related to type 2 diabetes mellitus self-management and adherence were subsequently applied to the development of a survey questionnaire, the CoMac Descriptor. ${ }^{9}$ The 12-question survey has a reading level at the sixthgrade level. The survey results categorize participants into eight different type 2 diabetes mellitus domains based on self-identification with actual words. The categories represent combinations of construct segments of high/low agency, positive/negative affect, and internal/external control orientation. Reliability and validity of the survey are presented in the previous research. ${ }^{5-8}$

The CoMac Descriptor Survey was used to segment the 72 patients in the intervention group into eight clusters that combine high/low agency, positive/negative emotion, and external/internal control orientation:

1. High agency/positive emotion/internal control (HPI),

2. High agency/positive emotion/external control (HPE),

3. Low agency/positive emotion/internal control (LPI),

4. High agency/negative emotion/internal control (HNI),

5. High agency/negative emotion/external control (HNE),

6. Low agency/positive emotion/external control (LPE),

7. Low agency/negative emotion/external control (LNE),

8. Low agency/negative emotion/internal control (LNI).

\section{Patient profile}

Prior to the implementation, the CoMac tools, which had been developed in basic research settings, needed adaptations for effective clinical use. The tools had to be adapted to provide HIPAA (Health Insurance Portability and Accountability Act)-compliant online survey segmentation with rapid analysis and prompt feedback. Therefore, an online grading system was developed to deliver immediate emailed segmentation results to the HCP, along with a onepage Patient Profile ${ }^{\mathrm{TM}}$. The Patient Profile includes the patient code, patient cluster, Points of Emphasis, and examples of Linguistic Cues. Figures 1 and 2 display sample patient profiles for HPI and LNE patient clusters, respectively. In addition, the three different colored backgrounds, green, yellow, and red, of the patient cluster "box" provide advice to the HCPs about patient orientation and potential adherence $^{7}$ : Green (as with the HPI cluster) denotes higher probability of adherence, yellow moderate adherence, and red lower adherence (as with the LNE cluster). The HCPs were educated in the interpretation of the color scheme before the study was conducted.

\section{Points of emphasis}

Included in the one-page Patient Profile, Points of Emphasis suggest language approaches that will be most effective for each patient in helping to achieve the desired outcome.

\section{Linguistic cues}

Also included in the Patient Profile, these provide specific examples of language that can be used in provider-patient communication.

\section{Data collection}

The data were collected in two phases. In Phase I, the implementation of the CoMac System was adapted to the clinic's standard patient flow. Specifically, the CoMac intervention was supported through additions to steps 2 and 5 as follows: 1) patients were referred to the clinic by local primary care physicians (PCPs) with the patients' HbAlc values taken within the last 90 days; 2) patients met with the diabetes team for an initial assessment session and completed the CoMac Descriptor Survey; 3) during the session, collaborative goal setting occurred using the AADE 7 Self-Care Behaviors; 4) patients met with the diabetes team in follow-up visits, with at least one follow-up visit occurring at least 30 days following the initial session; and 5) post-intervention $\mathrm{HbAlc}$ levels were 


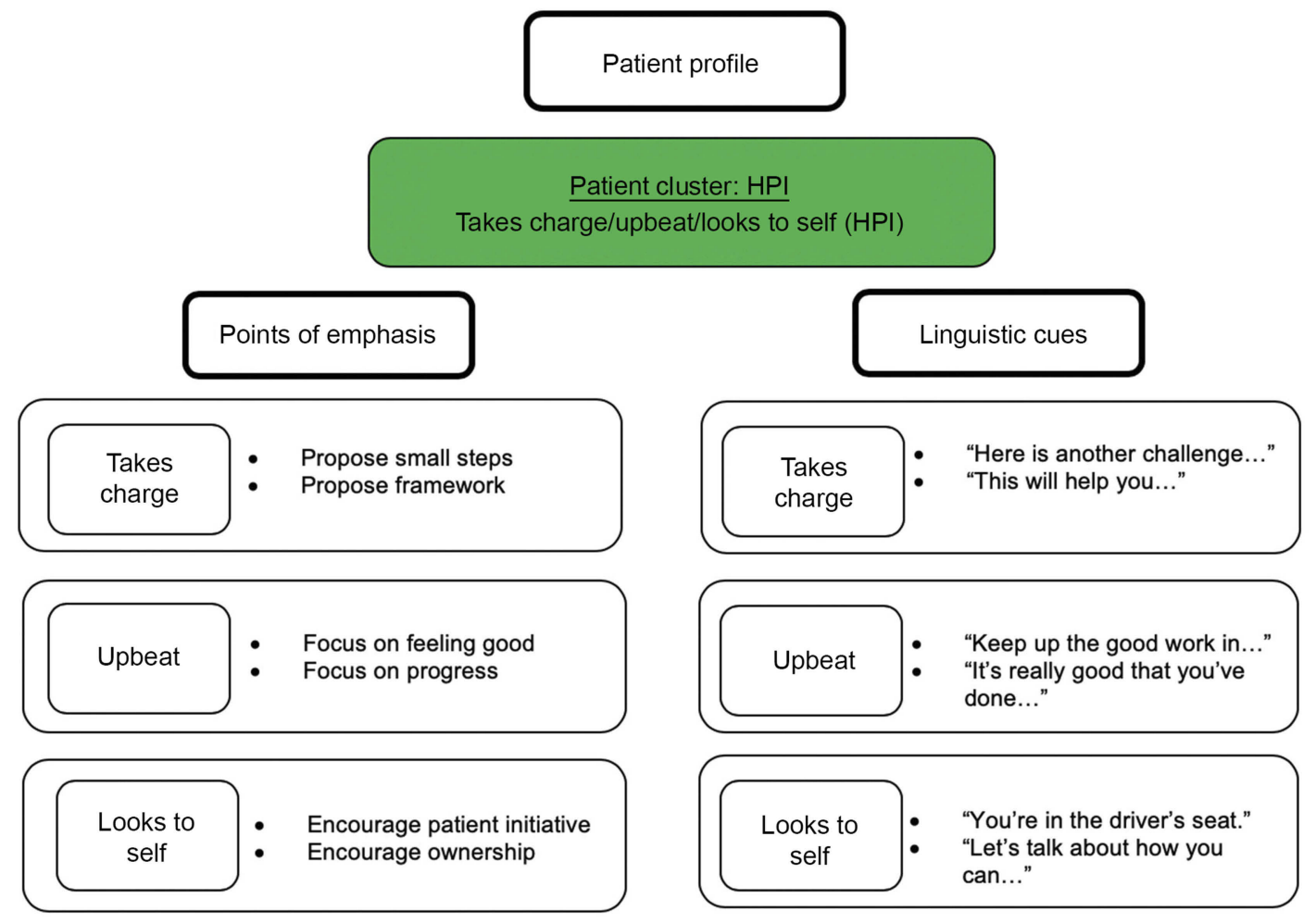

Figure I Sample patient profile (HPI).

Abbreviation: $\mathrm{HPI}$, high agency/positive emotion/internal control.

obtained from the PCP office electronic medical records. Qualitative, open-ended interview data were collected at monthly face-to-face meetings with the HCPs. Tool feasibility, patient and HCP satisfaction, and staff roles and responsibilities were the primary focus for Phase I.

In Phase II, patient outcomes were evaluated. To assess the impact of the intervention on patient outcomes, a preand post-intervention measure of $\mathrm{HbAlc}$ levels was collected along with other demographic and clinical data consistent with AADE requirements. Patient demographic characteristics including age, gender, starting weight, and type 2 or type 1 diabetes mellitus were gathered from the electronic medical record by the health care staff. Demographic characteristics were de-identified for use by the research team. Glycosylated hemoglobin (A1c) was gathered from the electronic medical record by the health care staff.

\section{Data analysis}

The interview notes obtained in Phase I were arranged thematically in a structure, process and outcome format. Rapid-cycle quality improvement (RCQI) was iteratively applied to identify barriers and adapt best practices in system implementation.
In Phase II, to analyze patient outcomes across the two groups, $t$-test and Chi-square statistics were calculated for between-group differences on demographic and HbA1c values. A multivariate regression analysis was performed to assess the predictors of change in $\mathrm{HbA} 1 \mathrm{c}$ by the intervention group. Analyses were conducted using Stata version 15 .

\section{Results and discussion}

To address the research questions stated previously, results and discussion will center both on the process of implementation and on patient outcomes.

\section{HCP qualitative feedback regarding CoMac intervention}

The process of the initial implementation suggested a minor alteration. To more securely survey patients, an iPad solely designated for the intervention, rather than a laptop computer, was used. Patients were familiar with the touch screen technology of the iPad and navigated the survey independently in most cases. From a technology and security perspective, the use of a laptop computer was awkward for patients and the team. Patients had difficulty with advancing screens, and an educator had to remain 


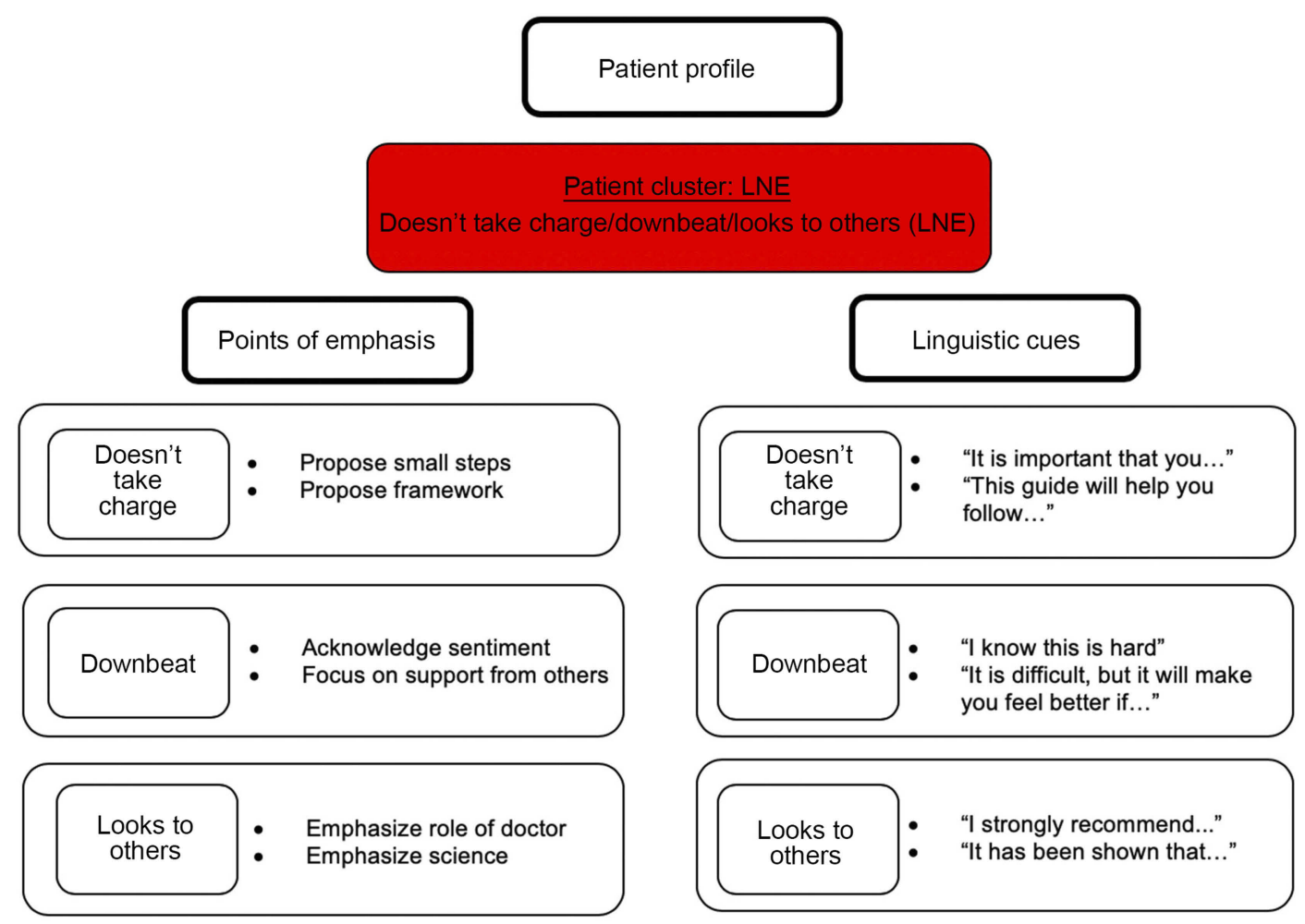

Figure 2 Sample patient profile (LNE).

Abbreviation: LNE, low agency/negative emotion/external control.

present during the survey since the tool was accessed through an employee's sign on.

The qualitative data from the HCP interviews produced the following key findings:

1. In diabetes education, the use of motivational interviewing to assess patient expectations and perceptions is considered the foundation for helping individuals. ${ }^{11}$ The CoMac System facilitated the timely collection of key information that is often challenging to gather and describe in motivational interviews. The tools were easy to use and added $<10$ mins to the initial assessment time. The completion of the Descriptor by the patient produced an immediate response to the team's distribution email account with the one-page Patient Profile.

2. The HCPs found the adherence prediction function of the Patient Profile (red, yellow, green color scheme) useful in suggesting an on-going self-management support plan. For instance, patients whose survey data suggested less likelihood of adherence were recommended for shorter follow-up periods, and follow-up appointments were scheduled prior to patients leaving the clinic.
3. The HCPs noted that Points of Emphasis and Linguistic Cues examples were valuable in engaging patients. One HCP commented, "The CoMac System helps [us] to get to the go point sooner" and "reveals potential barriers before the "dance." Another HCP stated, "As social media strategies evolve in chronic care management with patient portals, texting and email, the reliance on actual words that speak to individual patient's views will matter even more."

\section{Quantitative change in $\mathrm{HbAlc}$ pre- and post-intervention}

In addition to addressing the overall adaptability of the system, we were able to explore the impact of the CoMac System on patient outcomes between the intervention/treated and the control/nontreated groups.

As could have been expected by virtue of the clinic's effective education programming, all 120 patients showed improved clinical outcomes. The $t$ and $z$ tests listed here are all for one-variable comparisons across the intervention and control groups. The average weight loss for all the patients was more than 3 pounds: 3.1 pounds for the intervention group and 3.5 pounds for the control group 
(NS). The average HbAlc levels of the intervention group decreased from $9.0 \%(75 \mathrm{mmol} / \mathrm{mol})$ to $7.4 \%(57 \mathrm{mmol} /$ mol) (a decline of $1.6 \%(18 \mathrm{mmol} / \mathrm{mol})$ ). The $\mathrm{HbAlc}$ levels for the control group fell from $8.2 \%$ (66 mmol/ $\mathrm{mol})$ to $7.6 \%(60 \mathrm{mmol} / \mathrm{mol})$ (a decline of $0.6 \%$ (6 $\mathrm{mmol} / \mathrm{mol})$ ). Both declines are clinically meaningful, where clinically meaningful is defined as a decline of $0.5 \%$ or more. The fact that the post-intervention HbA1c level for the intervention group of $7.4 \%$ was below the level of the control at $7.6 \%$ suggests that the observed reductions in HbAlc were not simply reversions to the mean. Thus, even though the intervention group started with a higher average $\mathrm{HbAlc}$, their improvement was more than could be explained by chance or by the higher start. The greater reduction in the intervention group's average $\mathrm{HbA} 1 \mathrm{c}$ level is notable, as each $1 \%$ reduction in $\mathrm{HbA1c}$ levels represents significant relative risk reduction up to $26 \%$.

A multiple regression analysis predicting the change in $\mathrm{HbA1c}$ level showed that the independent variables of age, gender, and starting weight were not significant predictors of $\mathrm{HbA} 1 \mathrm{c}$ reduction. Table 2 shows the regression analysis output with the HbA1c change as the dependent variable, and all of the covariates in the multiple regression are listed therein. The starting value of $\mathrm{HbAlc}$ and the CoMac intervention were significant predictors of change in HbAlc at the follow-up time point. Patients with a higher initial HbAlc showed a greater decline in $\mathrm{HbAlc}$. The key variable of interest, treated with the CoMac intervention, had a $t$-value of $-1.81(P<0.04)$. These findings suggest that tailored communication associated with the use of the CoMac Communication System may result in short term and clinically meaningful improvements in HbA1c.

\section{Conclusion}

This article describes a pilot implementation of a psycholinguistic communication system, the CoMac Communication
System and tools, to individualize DSMES. This implementation research was designed to focus on the use of HCP appropriate language to match patient orientation to self-care behaviors. The study was conducted with the collaboration of the HCPs, with data collected in a clinical setting in a Midwestern US diabetes care clinic.

The CoMac System relies on the role of language to draw individuals' attention to health care messages. Selecting specific words and making linguistically informed adjustments to the way the words are used (eg, vocabulary, tone, sentence structure, and degree of directness) when communicating with people about self-management creates a psychological closeness between the message and the audience, also known as "verbal immediacy."12 Verbal immediacy facilitates the listener's connection to the message, which can increase one's attention to important information. This enhances understanding and learning, which is central to the art of DSMES. Indeed, increasing attention to the language of the spoken messages should be a key strategy in health communication to promote engagement and activation around diabetes self-management.

The HCPs in this study were able to implement the CoMac System and tools into their regular DSMES practice. Furthermore, qualitative interview and observation data, collected during the implementation, provided evidence about the potential positive impact of such language-centered communication on DSMES processes. According to the HCPs, an approach that combines a rigorous linguistic analysis of patient attitudes, combined with scientifically tested psychological predictors of patient behavior, leads to person-centered communication that can have a highly positive impact on the clinical process and the patient outcomes.

The HCPs welcomed the online CoMac System to supplement their existing procedures. Considering the fairly short period of this communication intervention, the significantly improved $\mathrm{HbAlc}$ levels of the intervention group are worth noting and encourage further investigations of the role of language in diabetes education.

Table 2 Regression output with HbAlc change as dependent variable

\begin{tabular}{|l|l|l|l|l|}
\hline Variable & Coefficient & Standard error & t & $\boldsymbol{P}$-value \\
\hline HbAlc start & -0.70 & 0.06 & -10.90 & 0.000 \\
CoMac Intervention & -0.42 & 0.23 & -1.81 & 0.037 \\
Age & 0.01 & 0.01 & 1.12 & 0.266 \\
Start weight & 0.00 & 0.00 & 1.10 & 0.274 \\
Male & -0.10 & 0.23 & -0.42 & 0.678 \\
\hline
\end{tabular}

Abbreviation: HbAlc, glycosylated hemoglobin Alc. 
This study was a pilot implementation study. As such, causality of findings cannot be established with the quasiexperimental design that was employed. Randomized controlled trials are needed to test the effectiveness of the use of the CoMac Communication System in a variety of diabetes education clinical settings. Data collection from this sample was limited to a convenience sample attending clinical care. Additional information about variables, such as the onset date of diagnosis, the timing of the HbAlc measurements, number of patient visits, other education interventions, and comorbidities, for instance, will provide an increased understanding about the role of language in patient education and self-care management.

Diabetes self-management is becoming more complex with each passing year. In response to the complexity, DSMES must become increasingly sophisticated to be effective. As the prevalence of diabetes continues to grow, health systems and educators will need tools to support DSMES management, risk stratification, and patient-centered interventions. ${ }^{11}$ In the collective voices of the HCPs who participated in the implementation research, "words do matter." The CoMac System fits with these expectations.

\section{Acknowledgments}

We gratefully acknowledge the cooperation of Columbus Regional Health Diabetes Services in the conduct of the study. The Diabetes Translational Research Center in the Indiana University School of Medicine provided invaluable assistance in the analysis of the data and manuscript preparation.

\section{Disclosure}

Dr Ulla Connor is the Chief Scientific Officer of CoMac Analytics, Inc. Dr Mary de Groot reports honoraria from Johnson \& Johnson Diabetes Institute, and provided ad hoc consultation funded internally for Eli Lilly, Inc., during the conduct of the study and outside the submitted work. Mr Robert Mac Neill is the Chief Executive Officer of CoMac Analytics, Inc. Dr Robert Sandy is the Principal of CoMac Analytics, Inc. The authors report no other conflicts of interest in this work.

\section{References}

1. Beck J, Greenwood D, Blanton L, et al. 2017 National standards for diabetes self-management education and support. Diabetes Care. 2017;40:1409-1419. doi:10.2337/dci17-0025

2. Dieleman JL, Baral R, Birger M, et al. US spending on personal health care and public health, 1996-2013. JAMA. 2016;316 (24):2627-2646. Published 2016 Dec 27. doi:10.1001/jama.2016. 16885

3. Riddle MC, Bakris G, Blonde L, et al. American Diabetes Association standards of medical care in diabetes - 2019. Diabetes Care. 2019;42(Suppl. 1):S34-S60. doi:10.2337/dc19-S004

4. Committee on Quality of Health Care in America, Institute of Medicine. Crossing the Quality Chasm: A New Health System for the 21st Century. Washington, DC: National Academies Press; 2001:1-22.

5. Connor U, Anton M, Goering E, et al. Listening to patients' voices: linguistic indicators related to diabetes self-management. Commun Med. 2012;9(1):1-12.

6. Connor U, Lauten K. A linguistic analysis of diabetes patients' talk: reported adherence to healthy behaviors. In: Hamilton HE, Chau WS, editors. The Routledge Handbook of Language and Health Communication. New York, NY: Routledge; 2014:91-108.

7. Sandy R, Connor U. Variation in medication adherence across patient behavioral segments: a multi-country study in hypertension. Patient Prefer Adherence. 2015;9:1539-1548. doi:10.2147/PPA.S91284

8. Bartlett Ellis RJ, Connor U, Marshall J. Development of patientcentric linguistically tailored psychoeducational messages to support nutrition and medication self-management in type 2 diabetes: a feasibility study. Patient Prefer Adherence. 2014;8:1399-1408. doi:10.2147/PPA.S69291

9. Connor U, Mac Neill RS, Mzumara HR, Sandy R. Development of the CoMac adherence descriptor: a linguistically-based survey for segmenting patients on their worldviews. Patient Prefer Adherence. 2015;9:509-515. doi:10.2147/PPA.S78178

10. Connor U, Kessler L, de Groot M, et al. CoMac communication system: a feasibility implementation of language-centered intervention in T2DM [abstract 251-OR]. Diabetes. 2018;67(Supplement 1):251-OR. doi:10.2337/db18-251-OR

11. Mensing CE, Cornell S, Halstenson C, et al. The Art and Science of Diabetes Self-Management Education Desk Reference. Chicago, IL: American Association of Diabetes Educators; 2014:110.

12. Parrott RL. Motivation to attend health messages: presentation of content and linguistic considerations. In: Maibach E, Parrott RL, editors. Designing Health Messages: Approaches from Communication Theory and Public Health Practice. Thousand Oaks, CA: Sage Publications, Inc.; 1995:7-24.
Patient Preference and Adherence

\section{Publish your work in this journal}

Patient Preference and Adherence is an international, peer-reviewed, open access journal that focusing on the growing importance of patient preference and adherence throughout the therapeutic continuum. Patient satisfaction, acceptability, quality of life, compliance, persistence and their role in developing new therapeutic modalities and compounds to optimize clinical outcomes for existing disease states are major areas of interest for the journal. This journal has been accepted for indexing on PubMed Central. The manuscript management system is completely online and includes a very quick and fair peer-review system, which is all easy to use. Visit http:// www.dovepress.com/testimonials.php to read real quotes from published authors. 